\title{
EDITORIAL
}

\section{Health in Context: COVID-19 Pandemic}

Syed Muhammad Imran Majeed, Rehma Ahsan Gilani

As the corona virus infection rates soar around the world, it remains to be seen whether the resurgent second wave will have the same fatality rate. The 1918-20 Spanish flu came in three waves, during which it killed at least 30 million people across the globe, with some historians quoting the figure at 100 million, making it more deadly than the total number of military and civilian deaths that resulted from World War.$^{1,2}$ The increase in lethality was assumed to be due to natural selection or random antigenic drift, accumulated by the virus in its initial first wave, that allowed the virus to evade existing immunity from previous infections. ${ }^{3}$ Korber et al. in their study has shown an amino acid change in the severe acute respiratory syndrome coronavirus 2 (SARS-CoV2) spike protein, D614G. This variant of SARS-CoV-2, containing G614, is now dominant in many places around the world. ${ }^{4,5}$ Based on the evidence collected from thousand COVID-19 cases in the United Kingdom, the authors have generated a hypothesis that reason for rapid spread of G614 is that it is more infectious than D614. ${ }^{4,5}$ Patients infected with viruses containing $\mathrm{G} 614$ had higher levels of virus RNA. ${ }^{5}$ In vitro experiments yielded high titers for G614 in pseudo viruses. ${ }^{6,7,8}$ However, implications of this preliminary data on the transmission patterns, disease presentation, vaccine and therapeutic development remain to be seen. Another aspect of this pandemic is the global focus upon breaking the chain of transmission since the cause of this crisis is viewed primarily as an infectious disease. But the story of COVID-19 is not so simple. Two categories of disease are interacting, infection with Severe Acute Respiratory Syndrome Coronavirus 2 (SARS-CoV-2) and Non-Communicable Diseases (NCDs). ${ }^{9}$ The clustering of these diseases within existing health inequalities among socially disadvantaged and low-income groups has amplified the adverse effects of each separate disease. Thus, COVID -19 is not just an epidemic ${ }^{10}$ but syndemic, a term coined by Merrill Singer, an American medical anthropologist. It is a synergistic epidemic characterized by aggregation of two or more concurrent disease clusters that adversely interact and affect each disease trajectory, resulting in an exacerbation of the prognosis and burden of disease. ${ }^{11}$ It appears that SARS CoV-2 patients in older age group, with chronic comorbidities like diabetes mellitus and hypertension and belonging to less advantage social strata racial and ethnic minorities, tend to suffer with more severe multisystem inflammatory syndrome. Therefore, successful containment of SARS-CoV-2 requires an urgent attention to NCDs and socioeconomic inequities. On a positive note, this pandemic has initiated a great human pause. The introspection, experienced during the lockdown, has made us review the very basics of the way we perceive and practice healthcare. It has made us wiser to execute our social contract by practicing a more socially conscious medicine. Today, in a post-COVID world, humanity stands at crossroads. In words of Robert Frost: "Two roads diverged in a wood, and I took the ones less travelled by, and that has made all the difference." ${ }^{12}$

\section{Editor-in-Chief}

\section{REFERENCE}

1. Phillips H. The recent wave of 'Spanish 'flu historiography. Social History of Medicine. 2014; 27: pp: 789-808.

2. https://www.history.com/news/spanish-flu-second-wave resurgence\#: :text= Mar\%203\%2C\%202020,Why \%20the\%20Second\%20Wave\%20of\%20the\%201918\%20Spanish\%20Flu\%20Was,the\%20fall\%20with\%20a\%20vengeance.\&text =Historians\%20now\%20believe\%20that\%20the,spread\%20by\%20wartime\%20troop\%20movements.

3. Viboud C, Tam T, Fleming D, Handel A, Miller M A, Simonsen L. Transmissibility and mortality impact of epidemic and pandemic influenza, with emphasis on the unusually deadly 1951 epidemic. Vaccine. 2006; 24: 6701-7.

4. Grubaugh ND, Hanage WP, Rasmussen AL. Making sense of mutation: what D614G means for the COVID-19 pandemic remains unclear. Cell. 2020; 182: 794-5.

5. Korber B, Fischer WM, Gnanakaran S, Yoon H, Theiler J, Abfalterer W, et al. Tracking changes in SARS-CoV-2 Spike: evidence that D614G increases infectivity of the COVID-19 virus. Cell. 182; pp: 812-27.

6. Lorenzo-Redondo R, Nam HH, Roberts SC, Simons LM, Jennings LJ, Qi C, et al. Ozer A Unique Clade of SARS-CoV-2 Viruses is 
Associated with Lower Viral Loads in Patient Upper Airways medRxiv. 2020.

7. Wagner C, Roychoudhury P, Hadfield J, Hodcroft E, Lee J, Moncla LH, et al. Comparing viral load and clinical outcomes in Washington State across D614G mutation in spike protein of SARS-CoV-2. 2020.

8. Hu J, He CL, Gao QZ, Zhang GJ, Cao XX, Long QX, et al. The D614G mutation of SARS-CoV-2 spike protein enhances viral infectivity and decreases neutralization sensitivity to individual convalescent sera bioRxiv. 2020.

9. Ozono S, Zhang Y, Ode H, Seng TT, Imai K, Miyoshi S, et al. Naturally mutated spike proteins of SARS-CoV-2 variants show differential levels of cell entry bioRxiv. 2020.

10. Horton R. Offline: COVID-19 is not a pandemic. Lancet (London, England). 2020; 396: 874.

11. Mendenhall E, Kohrt BA, Norris SA, detei DN, Prabhakaran D. Non-communicable disease syndemics: poverty, depression, and diabetes among low-income populations. Lancet. 2017; 389: 951-63.

12. Editorial The Lancet, Syndemics: health in context, Published. 2017; 389: P881. 